\title{
Ergonomic workplace analysis (EWA)
}

\author{
Deepak Sharan ${ }^{1}$ \\ ${ }^{1}$ RECOUP Neuromusculoskeletal Rehabilitation Centre, Bangalore, INDIA.
}

\begin{abstract}
Work Related Musculoskeletal disorders (WRMSDs) create a significant financial burden to both employer and employee that includes the cost of treatment and lost work time ${ }^{1}$. There is a growing demand to implement early identification and effective prevention strategies, aimed at early intervention in employees prior to possible absenteeism due to foreseen sickness or disability. A wide range of conditions may be considered as MSDs, including myofascial pain syndrome, fibromyalgia, peripheral entrapment neuropathies (carpal or cubital tunnel syndrome), tenosynovitis, epicondylitis, and others. Several multidisciplinary rehabilitation centres such as RECOUP have developed a comprehensive, systematic and thorough medical examination protocol for WRMSD patients. The aim is to obtain anatomically precise clinical findings as a guide to therapy. These assessment protocols typically includes Questionnaire data, Physical examination protocol, Special Examination tests and Laboratory tests. A methodical medical evaluation protocol produces a patient-specific list of symptoms and coordinated physical findings. Such protocols provide a sounder basis for developing a biomechanical and ergonomic explanation for the WRMSD patient's injuries and for planning and monitoring therapy. Importantly for the WRMSD patient, it also provides a detailed explanation of symptoms that are experienced and a logical plan for alleviating them. MWRSD patients should not be dismissed as having vague or non physiologic complaints without this type of evaluation. Objectives: This paper will discuss methods of identification of ergonomic risk factors among the workers engaged in video display terminal use which is practised at our tertiary level rehabilitation centre.
\end{abstract}

Keywords: Ergonomic Workplace Assessment, RULA, Workstation

\section{Ergonomic workplace assessment}

Based on the identified risk factors, the Ergonomic Workstation Assessment (EWA) was designed to include comprehensive information about various factors that may directly or indirectly affect WRMSD while working with computers. It consists of five sections. A snapshot of some of the sections is illustrated in Figure 1.

\section{Demographics}

Demographic data is very important before starting of any ergonomics study. Demographic data provides an idea about the population that is under consideration in any particular study. 'Demos' means population and 'graphy' means picture. The major elements in any demographic data are size, composition and distribution. Studies have shown that various demographic factors affect the prevalence rate of work related musculoskeletal disorders ${ }^{2}$. In the present Ergonomics Workstation Assessment apart from the information about age, height, weight and gender, the demographics section covers the information about hours of work per day, type of using video display (desktop/laptop), type of occupation, hours of telephone use, habit of exercise, daily physical activities, medical history and so on. To incorporate all these parameters in the demographic section helps in finding out the various occupational and non-occupational factors associated with development any musculoskeletal disorders.

\section{Workstation information}

Studies in literature reported that the height of the chair may affect the prevalence of musculoskeletal

Corresponding author, Email ID: deepak.sharan@recoup.in

${ }^{1}$ Address: \#312, 80 Feet Road, 10th Block, Further Extension of Anjanapura Layout, Bangalore-560062 $1051-9815 / 12 / \$ 27.50$ C 2012 - IOS Press and the authors. All rights reserved 
disorders among the workers using computer at their workstation $^{6}$. Apart from height of the chair keyboard placement, seating height, other devices of computers such as monitor, mouse, also affect the development of musculoskeletal disorders ${ }^{4,5}$. In this workstation evaluation consists of the ergonomic evaluation of the workstation components (seating, keyboard/input device, monitor, working surface). It further includes various dimensional measurements (i.e., chair height, eye-monitor distance) and questions regarding the workstation components (i.e., does the chair have armrests? keyboard tray being used? adequate leg room under table?). This section also includes information about environmental factors such as lighting, humidity, and temperature all these factors present in the workplace also has an effect on the development of musculoskeletal disorders.

\section{Working posture information}

Working posture has a significant effect on the severity, prevalence, incidence of work related musculoskeletal disorders in any of the workplace as well as at the computer workstations. In the present method the working posture is recorded using the modified version of Rapid Upper Limb Assessment (Leuder, 1996). The additional postural information is collected during the working condition i.e. while participant is working on the computer (i.e., head and neck in line with torso, forearm, wrist and hand in line).

\section{Perceived pain and discomfort}

The perceived pain section consists of ratings of perceived pain indicated by the participants for different body parts (neck, back, shoulder, elbows, wrists, knees, hips/thighs, ankles). This section consists of the Nordic pain score questionnaire along with ratings of pain on a scale of 1 to 5 . This section also consists of questions regarding numbness/discomfort while working and after the work is completely stopped.

\section{Workstyle information}

'Workstyle', or how a worker behaviourally, cognitively and physiologically responds to increased or stressful work demands, has been proposed to help explain the link between ergonomic and psychosocial factors in work-related upper limb disorder symptoms and disorders (WRULD). In the present method of evaluation a questionnaire developed by Feuerstein et $\mathrm{al}^{3}$ was used for evaluating the workstyle factors among the workers engaged in computer work. This questionnaire evaluates total ten factors such as Working Through Pain, Social Reactivity, Limited Workplace Support, Deadlines/Pressure, Self-imposed Workpace/ Workload, Breaks, Mood, Pain/Tension, Autonomic Response and Numbness/Tingling. The workstyle questionnaire consists of 32 questions and addresses psychosocial, physiological, and behavioral response that occurs in an individual due to high work.

\section{Implementation}

The EWA is currently being used by RECOUP as a part of workplace assessment services provided to the IT companies. After assessing the various factors observed, its Ergonomists provide recommendations in terms of adjustment of work components, posture awareness and correction, and treatment. So far, we have collected data from more than four thousand IT professionals and some of these data have been submitted to be published and will be available soon. Additionally, there are many on-going research projects which will utilize the data from the EWA. The EWA is continuously updated to include the most relevant information that may help in understanding the causes and progression of WRMSD. 


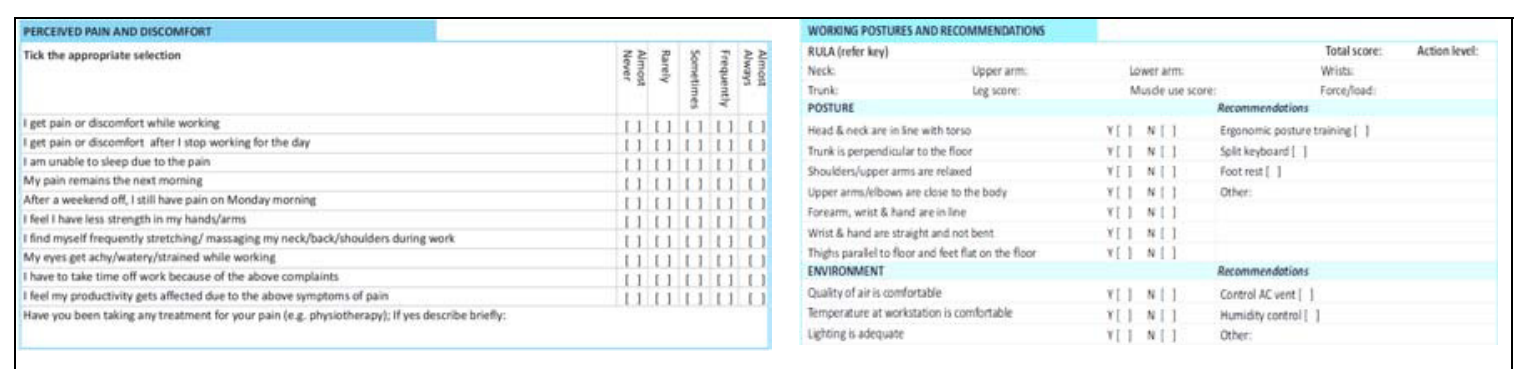

Figure 1. "Perceived pain and Discomfort" and "Working Posture Information" sections of the Ergonomic Computer Workstation Assessment.

\section{References}

[1] BLS. (2005). Nonfatal occupational injuries and illnesses requiring days away from work, 2005. Retrieved from www.bls.gov.

[2] J.W. Choi, Y.J.Kang, J.Y.Kim, S.H. Han, Symptoms prevalence of musculoskeletal disorders and the effect of prior acute injury among aging male steel workers, Journal of Occupational Health, 2009, 51, $273-282$.

[3] M. Feuerstein, R.A. Nicolas, G.D. Huang, A.J. Haufler, G . Pransky, M. Robertson, Work style: development of a measure of response to work in those with upper extremity pain, J Occup Rehabil, 2005, 15, 87-104.
[4] N.A Baker., E.L Cidboy., The Effect of Three Alternative Keyboard Designs on Forearm Pronation, Wrist Extension, and Ulnar Deviation: A MetaAnalysis, The American Journal of Occupational Therapy, 2006, 60, $40-49$.

[5] W. Swei-Pi, Y. Chien-Hsin, H. Cheng-Pin and J. DerHong , VDT Screen Height and Inclination Effects on Visual and Musculoskeletal Discomfort for Chinese Wheelchair Users with Spinal Cord Injuries, Industrial Health, 2009, 47, 89-93

[6] http://www.irsst.qc.ca 